\title{
CONDUCTANCE FLUCTUATIONS IN MICROSTRUCTURES OF HgCdMnTe BICRYSTALS
}

\author{
G. Grabecki, A. Lenard, W. Plesiewicz, J. Jaroszyński, \\ Marek Cieplak, T. Skośkiewicz, T. Dietl \\ Institute of Physics, Polish Academy of Sciences \\ Al. Lotników 32/46, 02-668 Warszawa, Poland
}

\begin{abstract}
E. Kamińska, A. Piotrowska
Institute of Electron Technology, Al. Lotników 32/46, 02-668 Warszawa, Poland
\end{abstract}

\author{
AND B. BULKA
}

Institute of Molecular Physics, Polish Academy of Sciences 60-179 Poznań, Poland

\begin{abstract}
Microscopic four-contact probes to semimagnetic $\mathrm{HgCdMnTe}$ grain-boundary inversion layers have been photolithographically patterned. Magnetoresistance measurements performed on these samples revealed aperiodic conductance fluctuations of the magnitude of the order of $e^{2} / h$. Quantitative analysis of both fluctuation amplitude and their mean period indicate that we have approached the mesoscopic regime in our system. This opens new possibilities in studies of spin-subsystem dynamics in semimagnetic semiconductors.
\end{abstract}

PACS numbers: $73.40 . \mathrm{Lq}$

It has recently been demonstrated that when linear size of the sample $L$ becomes smaller than the inelastic scattering length $L_{\text {in }}$, quantum interference of scattered waves gives rise to random but reproducible fluctuations of the conductance as a function of external parameters such as the magnetic field [2]. Theoretical calculations based on diagrammatic approach performed by Althsuler and Skhlovskii [1] and Lee at al. [2] predict the amplitude of conductance fluctuations for mesoscopic two-dimensional (2D) systems, $L<L_{\text {in }}$, to be $0.9 \mathrm{e}^{2} / h$. We have carried out numerical simulations of the conductance in such a system, which have given a similar value $0.7 e^{2} / h$ (see [3] for details). 
Since the above number depends only slightly on the symmetry of the system Hamiltonian (universality class) and, as long as $L<L_{\text {in }}$, it is entirely insensitive to $L$ and material parameters the phenomenon is known under the name of Universal Conductance Fluctuations (UCF). At the same time, the fluctuation pattern turns out to be extremely sensitive to the specific distribution of scattering centres in the measured sample. Therefore, experimental studies of these fluctuations in magnetic materials may be very perspective because the phenomenon of conductance fluctuations may serve as a valuable tool for probing complex dynamics of localized magnetic moments. In particular, one can study signatures of transition to the spin-glass phase $[4,5]$.

We have performed a study of microstructures obtained from $p-\mathrm{Hg}_{0.800} \mathrm{Cd}_{0.187}$ $\mathrm{Mn}_{0.013}$ Te bicrystals. The composition of the material has been established by means of electron microprobe. Magnetic susceptibility measurements have indicated that the spin-glass freezing transition occurs at $T=100 \mathrm{mK}$ in this material. As shown in our previous works [6], HgCdMnTe grain boundaries contain good quality, 2D electron inversion layers. In bicrystal used in the present study, 2D electron concentration was $n_{\mathrm{S}}=1 \times 10^{12} \mathrm{~cm}^{-2}$, and mobility $\mu=30000 \mathrm{~cm}^{2} / \mathrm{Vs}$. Our idea of resistance measurements involving a very small part of the grain boundary is based upon the local character of the four-probe method [7]. Thus, in order to probe a very small part of the bicrystal, we have made microcontacts to the grain boundaries by means of the photolithography and lift-off method. The scheme of our sample is illustrated in Fig. 1. Additional large contacts on the sample allowed simultaneous measurements in macro- and meso-scales. Besides, the same contact pattern has been made on the monocrystalline $\mathrm{HgCdMnTe}$ samples, cut from the same crystal. Resistance measurements performed on these samples at helium temperatures have shown values higher than $1 \mathrm{M} \Omega$, clearly indicating lack of shunting between closely spaced gold microcontacts.

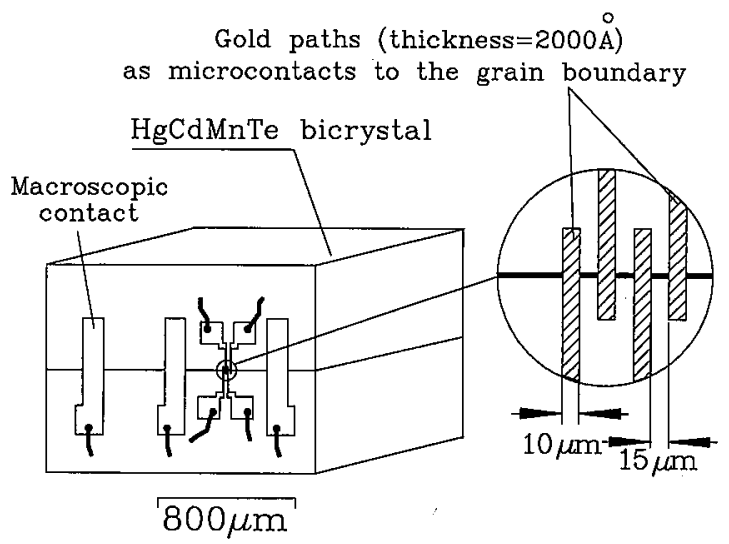

Fig. 1. HgCdMnTe bicrystal sample with photolithographically patterned contacts. Microcontacts to the grain boundary are shown in magnified scale.

An example of experimental results is presented in Fig. 2. In the case of 


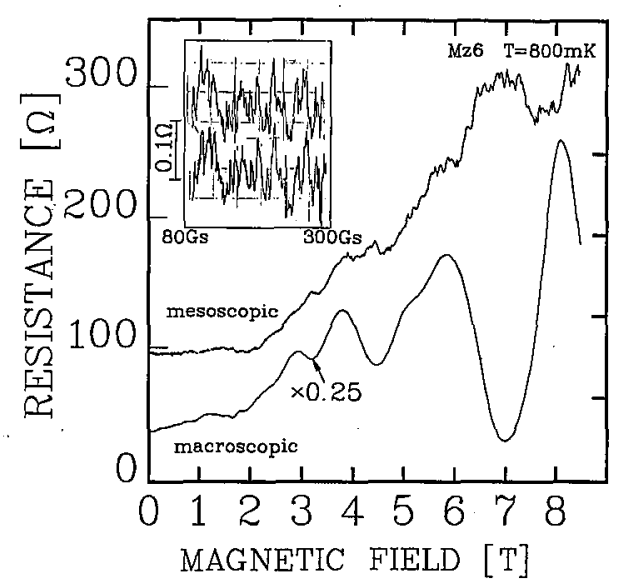

Fig. 2. Low-temperature magnetoresistance traces obtained for $\mathrm{HgCdMnTe}$ bicrystal on both the macro- and microcontact four-probes. Inset illustrates reproducibility of the fluctuations, observed during up and down magnetic field sweep at $35 \mathrm{mK}$.

macroscopic contacts the magnetoresistance exhibits smooth and pronounced Shubnikov-de Haas (SdH) oscillations. The deep minimum at $B=7 \mathrm{~T}$ is related to the quantum Hall effect in our 2D system. It should be noted that the SdH pattern in the magnetic fields $B<2 \mathrm{~T}$ has been found to be nearly the same for both macroand microcontacts and the measurements in tilted magnetic fields performed at $T=4.2 \mathrm{~K}$ have shown the $2 \mathrm{D}$ character of the magnetoresistance for both cases.

In contrast, the magnetoresistance measured on microcontacts at very low temperatures shows irregular fluctuations superimposed on the much less pronounced SdH oscillation pattern. Both the fluctuation amplitude and their typical spacing increase with the magnetic field. The inset to Fig. 2 illustrates a very good reproducibility of the aperiodic fluctuation pattern at $T=35 \mathrm{mK}$. From these results we have calculated root mean square of the conductance fluctuations amplitude $(\operatorname{rms}(g))$ which is about $0.25 e^{2} / h$ for $B<1 \mathrm{~T}$, whereas at $B=8 \mathrm{~T}$ it approaches $2.5 e^{2} / h$. The $\mathrm{rms}(g)$ is nearly constant at temperatures below $1 \mathrm{~K}$ and steeply decreases at higher temperatures (at $T=4.2 \mathrm{~K}$ its value is 4 times smaller than that at $800 \mathrm{mK}$ ). This decrease results from increasing role of inelastic scattering at high temperatures.

We interpret the observed phenomenon as the UCF. In order to examine whether such an interpretation is consistent with existing theories and other experimental data, one can perform analysis of the both fluctuation amplitude and their mean period. If one assumes that $L>L_{\text {in }}$, then the mean fluctuation period $\Delta B$ may be used for rough estimation of the coherence area $S \approx L_{\text {in }}^{2}$ of the measured sample, according to:

$$
\Delta B \approx \phi_{0} / S
$$

where $\phi_{0}=h / e$ is magnetic flux quantum. To obtain $\Delta B$ we have performed 
Fourier transformations of the fluctuation patterns. We have estimated $S$ to be between 10 and $100 \mu \mathrm{m}^{2}$ for fluctuations measured at $B<1 \mathrm{~T}$, whereas the data at $B=8 \mathrm{~T}$ give the values below $0.5 \mu \mathrm{m}^{2}$. Similar effect has been observed in quantum wires made of GaAs-AlGaAs heterostructures by Timp at al. [8], and interpreted in terms of magnetic field induced decrease in the width of the distribution of electron trajectories across the sample. Such an effect is possible only when the strong magnetic field quantization takes place. This gives us additional proof that the observed fluctuations originate from mobile 2D-electrons at the grain boundary.

If one calculates the $L_{\text {in }}$ from the low-field coherence area, one obtains value between 3 and $10 \mu \mathrm{m}$, considerably smaller than the probed region size (which should be as large as $75 \mu \mathrm{m}$ in our case, see Fig. 1). However, there are both the theoretical and experimental evidences that for certain sample geometries, the fluctuations may be significantly amplified even when the sample size exceeds considerably $L_{\text {in }}$. Lee at al. [2] have given expression for $\mathrm{rms}(\mathrm{g})$ in the case of a rectangular $L \times W 2 \mathrm{D}$ sample for which $W>L$ and the current flows along $\mathrm{L}$ :

$$
\operatorname{rms}(g)=e^{2} / h\left(L / L_{\text {in }}\right)^{1 / 2}\left(L_{\text {in }} / W\right)^{3 / 2} .
$$

In the macroscopic case, resistance measurement of a half-plane in the four-probe geometry give the same value as one performed on the rectangular sample made from the same material with the width 2.3 times greater than the length [9]. If one extends this analogy into mesoscopic case and substitutes into Eq. (2) the sample dimensions from Fig. 1: $L=25 \mu \mathrm{m}$ (the mean distance between voltage probes), $W=60 \mu \mathrm{m}$ (the mean distance between external contacts-analogous to the rectangle width) and $L_{\mathrm{in}}=2 \mu \mathrm{m}$ (the lower limit), then one obtains $\operatorname{rms}(g)$ $=0.125 e^{2} / h$ which is comparable favorably with our experimental data. In conclusion, the obtained results indicate that the studied system can be applied to elucidate various aspects of spin dynamics in the vicinity of spin-glass freezing.

\section{References}

[1] B.L. Al'thsuler, B.I. Skhlovskii, Zh. Eksp. Teor. Fiz. 91, 220 (1936).

[2] P.A. Lee, A. Douglas Stone, H. Fukuyarna, Phys. Rev. B 35, 1039 (1987).

[3] Marek Cieplak, B.R. Bulka, T. Dietl, to be published.

[4] B.L. Al'thsuler, B.Z. Spivak, Pis'ma Zh. Eksp. Teor. Fiz. 42, 363 (1985);

S. Feng, A.J. Bray, P. Lee, M.A. Moore, Phys. Rev. B 36, 5624 (1987).

[5] P.G.N. de Vegvar, L.P. Levy, T.A. Fulton, Phys. Rev. Lett 66, 2380 (1991).

[6] G. Grabecki, T. Suski, T. Dietl, T. Skośkiewicz, M. Gliński, in High Magnetic Fields in Semiconductor Physics, Ed. G. Landwehr, Springer-Verlag, Berlin, Heidelberg 1987, p. 127.

[7] M.M. Poljakov, Izw. Vuzov SSSR, Seria Fizika, No 1.0, 39 (1973).

[8] G. Timp, A.M. Chang, P. Mankiewich, R. Behringer, J.E. Cunningham, T.Y. Chang, R.E. Howard, Phys. Rev. Lett. 59,732 (1987).

[9] L.J. van der Pauw, Philips Res. Repts 13, 9 (1958). 